\title{
GRB 090313: X-shooter's first shot at a gamma-ray burst ${ }^{\star}$
}

A. de Ugarte Postigo ${ }^{1,2}$, P. Goldoni ${ }^{3,4}$, C. C. Thöne ${ }^{1}$, S. D. Vergani ${ }^{3}$, V. D’Elia ${ }^{5,6}$, S. Piranomonte ${ }^{5}$, D. Malesani ${ }^{7}$, S. Covino ${ }^{1}$, H. Flores ${ }^{8}$, J. P. U. Fynbo ${ }^{7}$, J. Hjorth ${ }^{7}$, R. A. M. J. Wijers ${ }^{9}$, S. D'Odorico ${ }^{10}$, F. Hammer ${ }^{8}$, L. Kaper ${ }^{9}$, P. Kjærgaard ${ }^{11}$, S. Randich ${ }^{12}$, M. I. Andersen ${ }^{7}$, L. A. Antonelli ${ }^{5}$, L. Christensen ${ }^{10}$, P. D'Avanzo ${ }^{1}$, F. Fiore ${ }^{5}$, P. J. Groot ${ }^{13}$, E. Maiorano ${ }^{14}$, E. Palazzi ${ }^{14}$, E. Pian ${ }^{15,16,10}$, G. Tagliaferri ${ }^{1}$, M. E. van den Ancker ${ }^{10}$, and J. Vernet ${ }^{10}$

1 INAF - Osservatorio Astronomico di Brera, via E. Bianchi 46, 23807 Merate, Lc, Italy e-mail: antonio.deugarte@brera.inaf.it

2 European Southern Observatory, Casilla 19001, Santiago 19, Chile

3 APC - UMR 7164, 10 rue Alice Domon et Léonie Duquet, 75205 Paris Cedex, France

4 Service d'Astrophysique, DSM/IRFU/SAp, CEA-Saclay, 91191 Gif-sur-Yvette, France

5 INAF - Osservatorio Astronomico di Roma, Via Frascati, 33, 00040, Monteporzio Catone (Rome), Italy

6 ASI - Science Data Centre, Via Galileo Galilei, 00044 Frascati (Rome), Italy

7 Dark Cosmology Centre, Niels Bohr Institute, University of Copenhagen, Juliane Maries Vej 30, 2100 Copenhagen $\emptyset$, Denmark

8 GEPI, Observatoire de Paris, CNRS, Université Paris Diderot, 5 place Jules Janssen, 92195 Meudon, France

9 Astronomical Institute, University of Amsterdam, Kruislaan 403, 1098 SJ Amsterdam, The Netherlands

10 European Southern Observatory, K. Schwarzschild Str. 2, 85748 Garching, Germany

11 Niels Bohr Institute, University of Copenhagen, Juliane Maries Vej 30, 2100 Copenhagen Ø, Denmark

12 INAF - Osservatorio Astrofisico di Arcetri, Largo E. Fermi 5, 50125 Firenze, Italy

13 Department of Astrophysics, IMAPP, Radboud University Nijmegen, PO Box 9010, 6500 GL Nijmegen, The Netherlands

14 INAF - IASF di Bologna, via Gobetti 101, 40129 Bologna, Italy

15 INAF - Osservatorio Astronomico di Trieste, via Tiepolo, 11, 34131 Trieste, Italy

16 Scuola Normale Superiore di Pisa, Piazza dei Cavalieri 7, 56126 Pisa, Italy

Received 12 August 2009 / Accepted 20 January 2010

ABSTRACT

Context. X-shooter is the first second-generation instrument to become operative at the ESO Very Large Telescope (VLT). It is a broad-band medium-resolution spectrograph designed with gamma-ray burst (GRB) afterglow spectroscopy as one of its main science drivers.

Aims. During the first commissioning night on sky with the instrument fully assembled, X-shooter observed the afterglow of GRB 090313 as a demonstration of the instrument's capabilities.

Methods. GRB 090313 was observed almost two days after the burst onset, when the object had already faded to $R \sim 21.6$. Furthermore, the $90 \%$ illuminated Moon was just 30 degrees away from the field. In spite of the adverse conditions, we obtained a spectrum that, for the first time in GRB research, simultaneously covers the range from 5700 to $23000 \AA$.

Results. The spectrum shows multiple absorption features at a redshift of 3.3736, which we identify as the redshift of the GRB. These features are composed of 3 components with different ionisation levels and velocities. Some of the features have never been observed before in a GRB at such a high redshift. Furthermore, we detect two intervening systems at redshifts of 1.8005 and 1.9597.

Conclusions. These results demonstrate the potential of X-shooter in the GRB field, because it was capable of observing a GRB down to a magnitude limit that would include $72 \%$ of long GRB afterglows $2 \mathrm{~h}$ after the burst onset. Coupled with the rapid response mode available at VLT, allowing reaction times of just a few minutes, X-shooter constitutes an significant leap forward on medium resolution spectroscopic studies of GRBs, their host galaxies and intervening systems, probing the early history of the Universe.

Key words. gamma-ray burst: general - instrumentation: spectrographs

\section{Introduction}

During the first hours after the onset of long gamma-ray bursts (GRBs, for a short-long classification see Kouveliotou et al. 1993), their optical/near infrared (nIR) counterparts shine as the brightest beacons in the Universe (Kann et al. 2007; Racusin et al. 2008; Bloom et al. 2009). Because they are generally produced by the collapse of a massive star (Woosley \& Bloom 2006, and references therein), they give us the opportunity to study the environment of massive star-forming regions at cosmological

^ Based on observations made with ESO VLT at Paranal Observatory during the commissioning of X-shooter. distances (the average redshift of long GRBs observed by Swift is 2.2; Fynbo et al. 2009) through the use of medium-to-high resolution spectroscopy. Time variability of absorption features corresponding to fine-structure and metastable level transitions has sometimes been identified (Dessauges-Zavadsky et al. 2006; Vreeswijk et al. 2007; D'Elia et al. 2009), which makes it possible to constrain the distance from the GRB to the absorbing gas, on typical scales of kpc. Only a few such cases have been spotted up to now, because of the required spectral resolution and sensitivity. Furthermore, as bright sources that disappear over time, they allow us to study intervening systems through their absorption signatures in the afterglow spectrum and to search 
for the associated galaxies when the afterglow has faded away (Jakobsson et al. 2004; Chen et al. 2009).

The family of short GRBs, although still poorly studied, presents fainter counterparts on average and it has been suggested that it is produced by the coalescence of compact objects (Nakar 2007, and references therein), although some of them could come from other progenitors, such as extragalactic magnetars (Hurley et al. 2005). Up to now, there have been very few attempts to acquire a spectrum of a short GRB, most of them were unsuccessful because of late observations or faint afterglows. There is still no medium or high-resolution spectrum of a short GRB.

Within this context, X-shooter (D'Odorico et al. 2006) is presented to the GRB community. It is the first of the secondgeneration instruments at ESO's Very Large Telescope (VLT) at Paranal Observatory (Chile). It is a single-target spectrograph capable of obtaining a medium-resolution spectrum $(\Re=4000$ 14000 , mainly depending on the slit width and wavelength) covering the complete range from 3000 to $24800 \AA$ in a single exposure. It has been designed to maximise efficiency by splitting the light with dichroics into three arms: ultraviolet/blue (UVB), visible (VIS), and near-infrared (NIR). Each arm has an echelle spectrograph with optimised optics, coatings, dispersive elements, and detectors. In March 2009, it was installed in the Cassegrain focus of Kueyen, the second $8.2 \mathrm{~m}$ Unit Telescope of the VLT, where it began routine scientific observations in October 2009.

One of the key science drivers of X-shooter is the study of optical and nIR afterglows of GRBs as cosmological probes of the circumstellar, interstellar, and intergalactic medium back to the epoch of first star formation in the Universe. During its first commissioning night on sky with its full setup, X-shooter was used to observe the afterglow of GRB 090313, which had exploded two days before, as a test target. In this article we present the results of this observation of a GRB with X-shooter, giving a hint of the full potential that the instrument will have during regular observations.

The paper is organised as follows. Section 2 describes our observations in the context of the GRB evolution. In Sect. 3 we present our results, focusing on the spectral features local to the GRB and on the intervening systems. Finally, in Sect. 4 we discuss the results and present our conclusions.

\section{Observations}

At 09:06:27 UT on 2009 March 13, Swift's Burst Alert Telescope (BAT) detected GRB 090313 (Mao et al. 2009), a long-duration burst with $T_{90}=78 \pm 19 \mathrm{~s}$ (Sakamoto et al. 2009). In response to the trigger, the $0.76 \mathrm{~m}$ Katzman Automatic Imaging Telescope (KAIT) slewed to the position shortly afterward and detected a bright ( $R \sim 16 \mathrm{mag}$ ) optical afterglow (Chornock et al. 2009a) at equatorial coordinates $(\mathrm{J} 2000.0): \mathrm{RA}=13^{\mathrm{h}} 13^{\mathrm{m}} 36^{\mathrm{s}} \cdot 21$, Dec $=$ $+08^{\circ} 05^{\prime} 49$ '. 2 (Updike et al. 2009).

The afterglow evolved through a plateau phase (Perley et al. 2009b; de Ugarte Postigo et al. 2009b), during which it maintained a magnitude of $I \sim 17.7$ up to almost a day after the burst, when the light curve steepened (de Ugarte Postigo et al. 2009c) and decayed following a power law $F \propto t^{-1.77}$ (Perley et al. 2009a). Perley (2009) used multicolour photometry to study the spectral energy distribution of the afterglow, concluding that its intrinsic extinction followed a Small Magellanic Cloud profile with a $V$-band extinction $A_{V} \lesssim 0.4$. This value was later refined by Kann et al. (2009) to $A_{V}=0.34 \pm 0.15$. They note that in the time interval between 0.02 and 0.5 days the afterglow

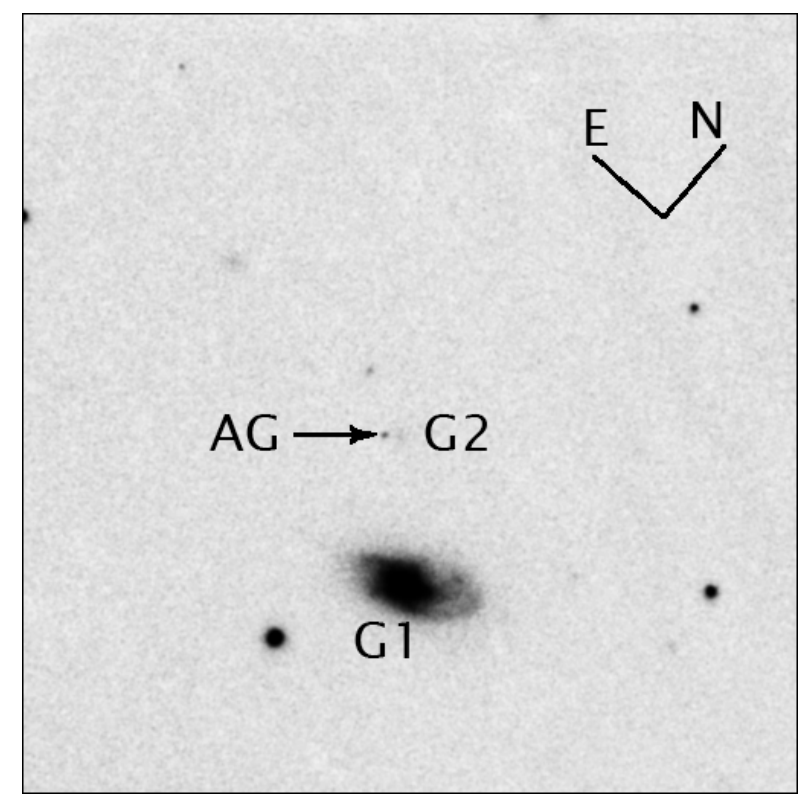

Fig. 1. I-band acquisition image. The GRB afterglow is indicated with an arrow. We also marked the two galaxies identified by Berger (2009), as mentioned in the discussion, G1 and G2 (G2 is faintly visible 2" NW of the afterglow). The figure shows the complete field of view of the acquisition and guiding camera, $90^{\prime \prime} \times 90^{\prime \prime}$. North and east are indicated in the figure.

of GRB 090313 was the optically brightest ever detected. A detailed multiwavelength study of the afterglow will be presented by Melandri et al. (in preparation).

Shortly after X-shooter came online for commissioning, it was aimed at the afterglow of GRB 090313. The observations consisted of a $900 \mathrm{~s}$ exposure and four additional exposures of $1500 \mathrm{~s}$ each, with a slit width of $0{ }^{\prime} 9$ in the VIS and NIR arms and $1^{\prime \prime} .0$ in UVB. The UVB and VIS detectors were used with $1 \times 2$ binning (binned in the spectral direction but not in the spatial one) and a slow readout of $100 \mathrm{kHz}$ to minimise the noise. The NIR detector was used in the default unbinned sample-up-theramp (non-destructive) mode. The resolution of the final spectrum varies with wavelength from 35 to $60 \mathrm{~km} \mathrm{~s}^{-1}$. All three spectral ranges were exposed during the first $900 \mathrm{~s}$. Inspection of this first set of frames showed a negligible signal in the UVB arm caused by strong contamination by the Moon, which was just 30 degrees away from the field and almost full (90\%). As a result, only VIS and NIR frames were obtained for the subsequent exposures. The mid-exposure time of the combined spectrum is 2009 March 15.26 UT, $45.1 \mathrm{~h}$ after the onset of the burst, when the afterglow had already faded to $R \sim 21.6$ (Perley et al. 2009a; Cobb 2009). On a $2 \times 20$ s combination of acquisition frames obtained at a mean time of $43.82 \mathrm{~h}$ after the burst, we measured $I=20.63 \pm 0.06$ (see Fig. 1). This magnitude is based on Cousin magnitudes derived from the SDSS catalogue ${ }^{1}$. The signal-to-noise ratio $(\mathrm{S} / \mathrm{N})$ per spectral bin of the spectrum varies with wavelength, reaching a maximum of $\sim 7$ around $8000 \AA$.

The data were reduced using a standard procedure with a preliminary version of X-shooter's pipeline (Goldoni et al. 2006, 2008). Because of variable conditions during the night of the observation, the spectra were not flux-calibrated, and a normalised spectrum is used in this work. No telluric correction was applied, so that prominent atmospheric bands (especially in the NIR) can

\footnotetext{
1 http://wWw . sdss . org/DR7/algorithms/ sdssUBVRITransform. html
} 
still be seen in Fig. 2, where we show the reduced normalised spectrum, which is a combination of all the individual spectra, covering the useful observing range (where the afterglow was detectable above the background level). The wavelengths reported throughout the paper are in vacuum. The uncertainty in the wavelength calibration is of the order of $30 \mathrm{~km} \mathrm{~s}^{-1}$ when using the current pipeline, which is the dominant uncertainty when calculating the redshift. For the analysis of the spectral features, we used FITLYMAN (Fontana \& Ballester 1995).

We caution that, because it was the first night of commissioning on sky, the instrument had not yet been optimised. Thus, the results presented here must not be considered as representing the optimal performance of the instrument but as a preliminary view of the potential that will be delivered by the instrument in regular operation. The results presented here supersede those reported by de Ugarte Postigo et al. (2009a).

\section{Results}

Over the complete range of the spectrum, we identify a main absorption system that is composed of several velocity components. To determine the central redshift of the absorption system, the spectra were rebinned such that the resulting absorption line centre represents the wavelength where the strongest absorption takes place. By excluding the blended absorption

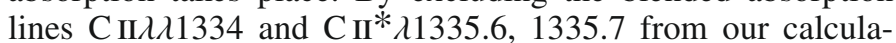
tions (see Sect. 3.1), we derive a redshift of $3.3736 \pm 0.0004$, which we identify as the redshift of the GRB. This value is consistent with the one previously determined by Chornock et al. (2009b) using GMOS/Gemini-South and Thöne et al. (2009) with FORS2/VLT.

Furthermore, we identify two intervening systems in the spectrum, one of which can again be divided into several velocity components. We determine the redshift in the same way as for the host galaxy system and derive redshifts of $1.9597 \pm 0.0003$ for the higher redshift system and $1.8005 \pm 0.0003$ for the multicomponent lower redshift system. Figure 2 shows the complete normalised X-shooter spectrum, where the absorption features of both the GRB and the intervening absorption systems are marked. The rest frame equivalent widths $\left(E W_{\text {rest }}\right)$ of all identified features are listed in Table 1, together with their observed and rest frame wavelengths and redshift. All wavelengths are in vacuum. For homogeneity, the values for all the velocity components are combined together in the table, as only some of them could be measured independently. We have also divided the table into three parts with the host redshift lines, intervening system at $z=1.96$ and intervening system at $z=1.80$.

\subsection{The host galaxy system}

Most of the absorption features detected at the redshift of the GRB can be separated into three different components that we name I, II, and III, where I has the highest redshift. We fit line profiles to the different components in order to determine the column density of each species. Because of the limited resolution and $\mathrm{S} / \mathrm{N}$, we fix the thermal $b$-parameter to $5 \mathrm{~km} \mathrm{~s}^{-1}$ and the turbulent $b$-parameters to $20 \mathrm{~km} \mathrm{~s}^{-1}$ (values that give the best global result for the fits), leaving only the column density as a free parameter. Transitions of the same element and ionisation level were fitted with a common column density. Some transitions are not well-fitted with only three components, which might indicate that there are other, smaller components not resolved in our spectrum. Furthermore, some of the lines are clearly saturated, so that only lower limits of the column densities can be derived for
Table 1. List of features identified in the spectra (detections $\gtrsim 3-\sigma$ ) along with their rest-frame equivalent widths.

\begin{tabular}{|c|c|c|c|}
\hline $\begin{array}{l}\lambda_{\text {obs }} \\
(\AA)\end{array}$ & $\begin{array}{c}\text { Feature } \\
(\AA)\end{array}$ & $z$ & $\begin{array}{c}E W_{\text {rest }} \\
(\AA)\end{array}$ \\
\hline \multicolumn{4}{|c|}{ Host galaxy at $z=3.37$} \\
\hline 5695.71 & О І $\lambda 1302.17$ & 3.37402 & $0.36 \pm 0.11$ \\
\hline 5705.96 & $\begin{array}{l}\text { Si II } \lambda 1304.37 \\
\text { C II } \lambda 1334.53\end{array}$ & 3.37450 & $0.58 \pm 0.12$ \\
\hline 5839.99 & $\begin{array}{l}\text { C II* }{ }^{*} \lambda 1335.66 \\
\mathrm{C}_{\text {III }} * \lambda 1335.71\end{array}$ & 3.37413 & $0.88 \pm 0.12$ \\
\hline 6096.13 & Si IV $\lambda 1393.75$ & 3.37389 & $1.14 \pm 0.13$ \\
\hline 6135.08 & Si IV $\lambda 1402.77$ & 3.37354 & $1.03 \pm 0.10$ \\
\hline 6678.24 & Si II $\lambda 1526.71$ & 3.37428 & $0.95 \pm 0.08$ \\
\hline 6770.31 & C IV $\lambda 1548.19$ & 3.37303 & $0.91 \pm 0.07$ \\
\hline 6781.67 & C IV $\lambda 1550.77$ & 3.37310 & $0.89 \pm 0.06$ \\
\hline 7035.95 & Fe II $\lambda 1608.45$ & 3.37437 & $0.62 \pm 0.06$ \\
\hline 7308.47 & Al II $\lambda 1670.79$ & 3.37427 & $1.29 \pm 0.06$ \\
\hline 8111.69 & Al III $\lambda 1854.72$ & 3.37355 & $0.71 \pm 0.04$ \\
\hline 8146.86 & Al III $\lambda 1862.79$ & 3.37347 & $0.56 \pm 0.05$ \\
\hline 9019.61 & Cr II $\lambda 2062.23$ & 3.37371 & $0.14 \pm 0.02$ \\
\hline 10385.9 & Fe II $\lambda 2374.46$ & 3.37401 & $1.12 \pm 0.10$ \\
\hline 10420.9 & Fe II $\lambda 2382.77$ & 3.37348 & $1.11 \pm 0.17$ \\
\hline 11312.1 & Fe II $\lambda 2586.65$ & 3.37326 & $1.21 \pm 0.49$ \\
\hline 11347.5 & Mn II $\lambda 2594.74$ & 3.37327 & $1.04 \pm 0.14$ \\
\hline 11372.2 & Fe II $\lambda 2600.17$ & 3.37363 & $1.29 \pm 0.11$ \\
\hline 12233.7 & Mg II $\lambda 2796.35$ & 3.37486 & $1.94 \pm 0.15$ \\
\hline 12267.9 & $\mathrm{Mg}$ II $\lambda 2803.53$ & 3.37588 & $1.90 \pm 0.13$ \\
\hline 12476.5 & $\mathrm{Mg} \mathrm{I} \lambda 2852.96$ & 3.37317 & $0.51 \pm 0.05$ \\
\hline 17207.2 & Ca II $\lambda 3934.78$ & 3.37311 & $1.21 \pm 0.06$ \\
\hline 17359.6 & Сa II $\lambda 3969.59$ & 3.37314 & $0.45 \pm 0.04$ \\
\hline \multicolumn{4}{|c|}{ Intervening system at $z=1.96$} \\
\hline 6937.93 & Fe II $\lambda 2344.21$ & 1.95960 & $0.15 \pm 0.04$ \\
\hline 7052.26 & Fe II $\lambda 2382.77$ & 1.95969 & $0.18 \pm 0.04$ \\
\hline 7695.64 & Fe II $\lambda 2600.17$ & 1.95966 & $0.31 \pm 0.04$ \\
\hline 8276.48 & Mg II $\lambda 2796.35$ & 1.95974 & $0.50 \pm 0.03$ \\
\hline 8297.48 & $\operatorname{Mg}$ II $\lambda 2803.53$ & 1.95965 & $0.59 \pm 0.05$ \\
\hline \multicolumn{4}{|c|}{ Intervening system at $z=1.80$} \\
\hline 6673.45 & Fe II $\lambda 2382.77$ & 1.80072 & $0.34 \pm 0.05$ \\
\hline 7240.68 & Fe II $\lambda 2586.65$ & 1.79925 & $0.48 \pm 0.06$ \\
\hline 7280.21 & Fe II $\lambda 2600.17$ & 1.79989 & $0.67 \pm 0.07$ \\
\hline 7830.87 & Mg II $\lambda 2796.35$ & 1.80039 & $1.35 \pm 0.10$ \\
\hline 7849.07 & $\operatorname{Mg}$ II $\lambda 2803.53$ & 1.79970 & $1.31 \pm 0.08$ \\
\hline 7987.58 & $\mathrm{Mg} \mathrm{I} \lambda 2852.96$ & 1.79974 & $0.48 \pm 0.08$ \\
\hline
\end{tabular}

them. The results of the fits are displayed in Table 2. Transitions marked with brackets in the table are contaminated by skylines or blended, and have not been used to fit the column densities. Upper limits are 3- $\sigma$, where the wavelengths were fixed to the corresponding components observed in other transitions. For saturated features we give lower limits for the column density. The fits to the highest $\mathrm{S} / \mathrm{N}$ or least saturated transitions for each ion are shown in Fig. 3.

The exact number of components for $\mathrm{Mg}$ II is not known because of the contamination with skylines so we fitted just the outer components I and III. Moreover, saturation meant that we could derive only lower limits for the column densities of these components. For Mn II, we could not fit component I owing to contamination by skylines. The absorption bluewards of component III in Si II $\lambda 1526$ is produced by Fe II $\lambda 2382$ from the intervening system at redshift 1.80 . We did not fit the OI $\lambda 1302$ transition because of the low $\mathrm{S} / \mathrm{N}$ at the blue end of the spectrum. For weaker lines where we did not detect all three components, we give 3- $\sigma$ upper limits for those components unless those regions are affected by skylines or other absorption transitions. 


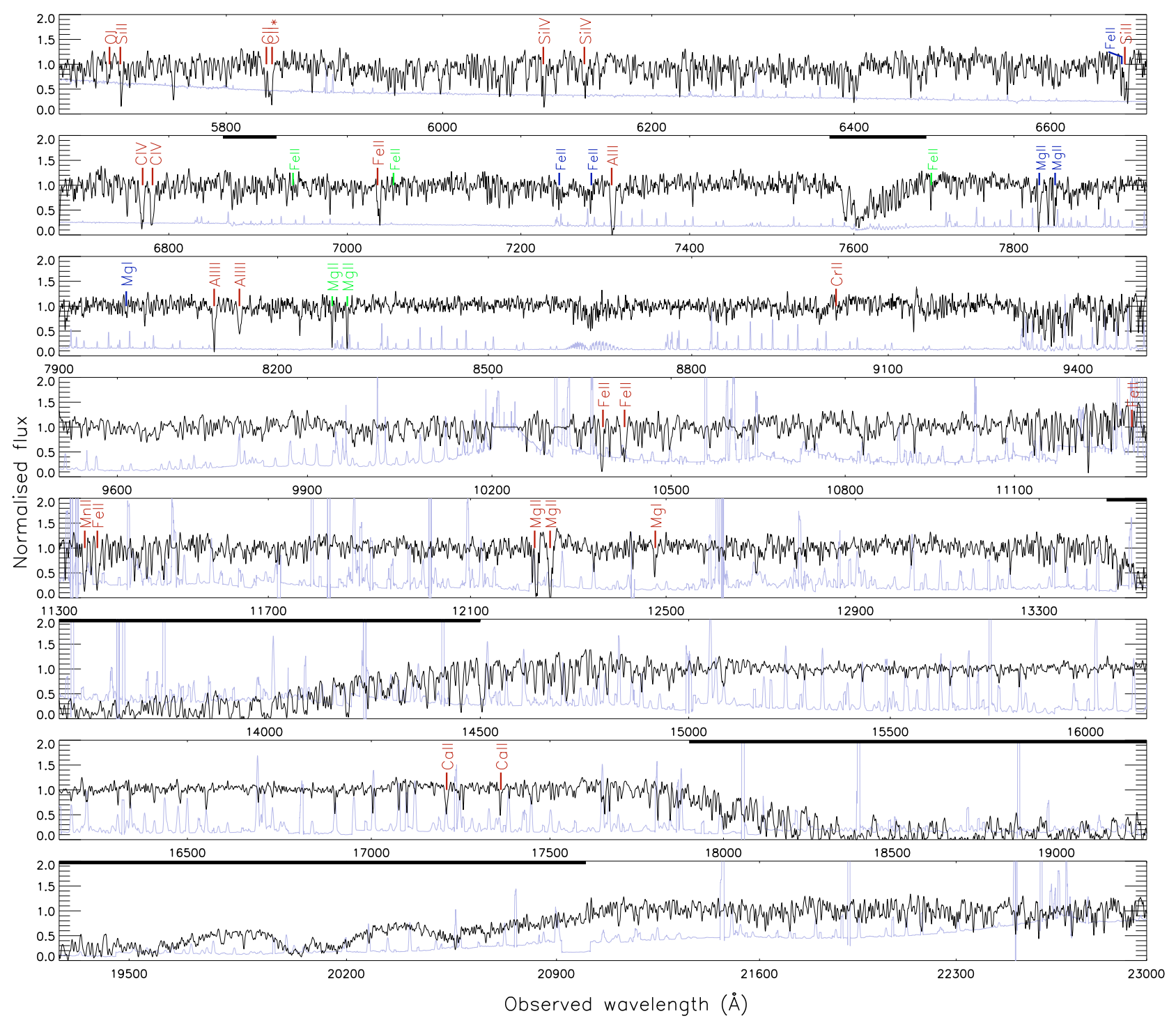

Fig. 2. Normalised spectrum of the afterglow of GRB 090313, smoothed with a Gaussian filter (using a smoothing kernel with full width half maximum varying from 1 to $4 \AA$ ) for displaying purposes. The absorption features produced at the redshift of the GRB afterglow ( $z=3.3736)$ are indicated in larger font size (red). The intervening systems at $z=1.8005$ and $z=1.9597$ are indicated in lower case and using dark (blue) and light (green) traces, respectively. The thick horizontal lines at the top of each panel indicate regions affected by strong telluric bands, which have been excluded from the analysis. The error spectrum is marked as a pale (blue) line. See the electronic edition for a colour version of this figure.

The central component II is the one with the highest column density and, except for Fe II, it could be fixed to $0 \mathrm{~km} \mathrm{~s}^{-1}$. The blueshifted component III is around $-85 \mathrm{~km} \mathrm{~s}^{-1}$ for all elements where this component is present except for Fe II where it lies at $-47 \mathrm{~km} \mathrm{~s}^{-1}$. For component I, the situation is slightly more complex. Al II, Al III, and Fe II have component I at $+102 \mathrm{~km} \mathrm{~s}^{-1}$, whereas it is at $+130 \mathrm{~km} \mathrm{~s}^{-1}$ for the Si lines, as well as for Mg II. Components I and III are absent for the weak lines Ca II, Cr II, and $\mathrm{Zn}$ II. For Mg I we only detect components I and II, whereas for C IV we only detect components II and III. Fe II has 3 components but at slightly different velocities than the other lines. We cannot confirm this behaviour securely by fitting other Fe II lines, since the $\lambda 1608 \AA$ line is the only one not severely affected by skylines. This behaviour of the different components might indicate that the absorbing material is not at the same place along the line-of-sight for different elements, which is expected if the absorption lines trace gas at different locations inside the host galaxy. Generally, we find that in component I the low ionisation species predominate over the higher ionisation species, while for component III the opposite is true. This is also confirmed by the ratio of column densities of $\mathrm{Al} \mathrm{III} \mathrm{and} \mathrm{Al}$ II for which we get values of $0.03 \pm 0.03,0.14 \pm 0.13$, and $4.2 \pm 0.9$ for components I, II, and III, respectively. A similar behaviour is observed for the ratio of column densities of Si IV and Si II with values of $0.02 \pm 0.01$, $0.91 \pm 0.26$, and $1.18 \pm 0.14$ for components I to III.

We detect the fine structure transitions of C II $\lambda 1334$, C II* $\lambda 1335.6,1335.7$. The last two only have a $0.1 \AA$ difference in central wavelength, so cannot be separated with the spectral resolution provided by X-shooter. However, the $1335.7 \AA$ transition is 10 times stronger than the $1335.6 \AA$ one, leading us to fit the absorption systems assuming that the stronger transition is responsible for most of the absorption. The resonant and finestructure transitions are separated well enough to allow a fit of all 3 components for both lines. Only component III of the fine structure transition is rather close to component I of the resonant line, making the system blended. On the other hand, we cannot identify any Si II* lines at the redshift of the GRB, which were 
A. de Ugarte Postigo et al.: GRB 090313: X-shooter's first shot at a gamma-ray burst

Table 2. List of features identified in the spectrum (detections $\gtrsim 3-\sigma$ ).

\begin{tabular}{cccccccc}
\hline \hline & \multicolumn{2}{c}{ Component I } & \multicolumn{2}{c}{ Component II } & \multicolumn{2}{c}{ Component III } \\
\hline Ion & $\begin{array}{c}\text { Transitions } \\
(\AA)\end{array}$ & $\begin{array}{c}\text { Velocity } \\
\left(\mathrm{km} \mathrm{s}^{-1}\right)\end{array}$ & $\begin{array}{c}\log N \\
\left(\mathrm{~cm}^{-2}\right)\end{array}$ & $\begin{array}{c}\text { Velocity } \\
\left(\mathrm{km} \mathrm{s}^{-1}\right)\end{array}$ & $\begin{array}{c}\log N \\
\left(\mathrm{~cm}^{-2}\right)\end{array}$ & $\begin{array}{c}\text { Velocity } \\
\left(\mathrm{km} \mathrm{s}^{-1}\right)\end{array}$ & $\begin{array}{c}\log N \\
\left(\mathrm{~cm}^{-2}\right)\end{array}$ \\
\hline Si IV & 1393,1402 & +130 & $13.3 \pm 0.3$ & 0 & $>15.3$ & -85 & $13.8 \pm 0.3$ \\
Si II & 1526 & +130 & $15.0 \pm 0.5$ & 0 & $>15.4$ & -85 & $13.7 \pm 0.2$ \\
C IV & 1548,1550 & +102 & $13.2 \pm 0.3$ & 0 & $>15.2$ & -85 & $>14.5$ \\
C II & 1334 & +102 & $14.1 \pm 0.4$ & 0 & $14.2 \pm 0.4$ & -85 & $15.3 \pm 0.6$ \\
C II & 1335 & +102 & $15.3 \pm 0.8$ & 0 & $14.4 \pm 0.4$ & -85 & $13.7 \pm 0.5$ \\
Fe II & $1608,(2374)$ & +102 & $14.9 \pm 0.2$ & +10 & $14.5 \pm 0.1$ & -47 & $13.9 \pm 0.2$ \\
Al III & 1854,1862 & +102 & $12.8 \pm 0.1$ & 0 & $14.4 \pm 0.2$ & -85 & $13.1 \pm 0.1$ \\
Al II & 1670 & +102 & $>14.6$ & 0 & $>15.4$ & -85 & $12.5 \pm 0.2$ \\
Cr II & 2062 & - & - & 0 & $13.6 \pm 0.1$ & $(-85)$ & $<13.6$ \\
Mg II & $2796,(2803)$ & +130 & $>14.8$ & - & - & -85 & $>13.6$ \\
Mg I & 2853 & +130 & $12.2 \pm 0.3$ & 0 & $13.1 \pm 0.1$ & $(-85)$ & $<12.6$ \\
Mn II & 2594 & - & - & 0 & $>14.3$ & -85 & $>13.6$ \\
Ca II & $(3933), 3969$ & $(+102)$ & $<12.6$ & 0 & $13.3 \pm 0.4$ & $(-85)$ & $<13.0$ \\
\hline
\end{tabular}

Table 3. List of column densities measured for the transitions identified in the $z=1.96$ intervening system.

\begin{tabular}{cccc}
\hline \hline & & \multicolumn{2}{c}{ Component I } \\
\hline Ion & $\begin{array}{c}\text { Transitions } \\
(\AA)\end{array}$ & $\begin{array}{c}\text { Velocity } \\
\left(\mathrm{km} \mathrm{s}^{-1}\right)\end{array}$ & $\begin{array}{c}\log N \\
\left(\mathrm{~cm}^{-2}\right)\end{array}$ \\
\hline Fe II & $2344,2382,2600$ & 0 & $\begin{array}{c}14.9 \pm 0.5 \\
\mathrm{Mg} \text { II }\end{array}$ \\
2796,2803 & 0 & $>15.5$ \\
\hline
\end{tabular}

Table 4. List of column densities measured for the transitions identified for the 3 different velocity components in the $z=1.80$ intervening system.

\begin{tabular}{cccccccc}
\hline \hline & & \multicolumn{2}{c}{ Component I } & \multicolumn{2}{c}{ Component II } & \multicolumn{2}{c}{ Component III } \\
\hline Ion & $\begin{array}{c}\text { Transitions } \\
(\AA)\end{array}$ & $\begin{array}{c}\text { Velocity } \\
\left(\mathrm{km} \mathrm{s}^{-1}\right)\end{array}$ & $\begin{array}{c}\log N \\
\left(\mathrm{~cm}^{-2}\right)\end{array}$ & $\begin{array}{c}\text { Velocity } \\
\left(\mathrm{km} \mathrm{s}^{-1}\right)\end{array}$ & $\begin{array}{c}\log N \\
\left(\mathrm{~cm}^{-2}\right)\end{array}$ & $\begin{array}{c}\text { Velocity } \\
\left(\mathrm{km} \mathrm{s}^{-1}\right)\end{array}$ & $\begin{array}{c}\log N \\
\left(\mathrm{~cm}^{-2}\right)\end{array}$ \\
\hline $\mathrm{Fe}$ II & $2382,2586,2600$ & +85 & $13.3 \pm 0.1$ & 0 & $15.0 \pm 0.2$ & -286 & $14.0 \pm 0.1$ \\
$\mathrm{Mg}$ II & 2796,2803 & +85 & $13.4 \pm 0.1$ & 0 & $>15.3$ & -286 & $>14.7$ \\
$\mathrm{Mg}$ I & 2853 & +85 & $12.3 \pm 0.2$ & 0 & $12.0 \pm 0.2$ & -286 & $12.5 \pm 0.2$ \\
\hline
\end{tabular}

reported for this GRB by Chornock et al. (2009b). However, our spectrum only covers the $1533.4 \AA$ and the $1309.2 \AA$ features and not that at $1264.7 \AA$, which is significantly stronger. We determined a 3- $\sigma$ upper limit for Si II ${ }^{*} \lambda 1533.4$ of $E W_{\text {rest }}<0.21 \AA$ and a limit for $\mathrm{Si}$ II ${ }^{*} \lambda 1309.2$ of $E W_{\text {rest }}<0.58 \AA$, the first being more constraining due to both the higher oscillator strength and $\mathrm{S} / \mathrm{N}$.

Combining the column density of all three components, we find a value of $[\mathrm{Si} / \mathrm{Fe}]>0.65$ for the GRB host galaxy (reference solar composition obtained from Asplund et al. 2005). This high value of $[\mathrm{Si} / \mathrm{Fe}]$ agrees with the work of Prochaska et al. (2007) for GRBs, who obtains higher values than those found for intervening absorbers in quasar spectra.

\subsection{Intervening systems}

An intervening system is identified at a redshift of 1.9597 through the detection of Fe II and Mg II in absorption (see Fig. 4 and Table 3).

At a redshift of 1.8005 we detect a further intervening system through several absorption features produced by low ionisation transitions (Fe II and Mg II), as well as neutral MgI. This system is formed by three components with relative velocities of $-286 \mathrm{~km} \mathrm{~s}^{-1}, 0 \mathrm{~km} \mathrm{~s}^{-1}$, and $+85 \mathrm{~km} \mathrm{~s}^{-1}$ (see Fig. 5 and Table 4).
The absorber at $z=1.8$ can be classified as a strong absorber $\left(\mathrm{Mg}\right.$ II $\lambda 2796 E W_{\text {rest }}>1 \AA$, see Table 1). Prochter et al. (2006) points out an excess (by a factor of $\sim 4$ ) in the frequency of strong Mg II $\lambda 2796$ absorbers in the line of sight of GRBs as compared to quasars. Recently, Vergani et al. (2009), have used a sample of GRB afterglow spectra that doubles the redshift path of previous studies to confirm this excess but only by a factor of $\sim 2$. The comparison is done using the SDSS QSO survey with a redshift range $0.37<z<2.27$ and a $6-\sigma$ detection limit of the $\mathrm{Mg}$ II $\lambda 2796$ line. Following these criteria, the redshift path of the X-shooter spectrum of GRB 090313 for strong systems is $\Delta z=1.13$, giving a number density of 0.88 . This number density agrees with that of $0.70 \pm 0.15$ found by Vergani et al. (2009), whereas the number density of the strong $\mathrm{Mg}$ II systems found along QSO lines of sight is only 0.28 \pm 0.01 (Nestor et al. 2005).

\section{Discussion and conclusions}

In this paper we present the first spectrum of a GRB obtained with X-shooter, covering a range from optical to NIR simultaneously for the first time. Although observed during an early commissioning phase and under unfavourable conditions, the results allow us to have an optimistic view of X-shooter's potential.

For the GRB, we determined a redshift of 3.3736, at which we detect absorption features with three velocity components. 


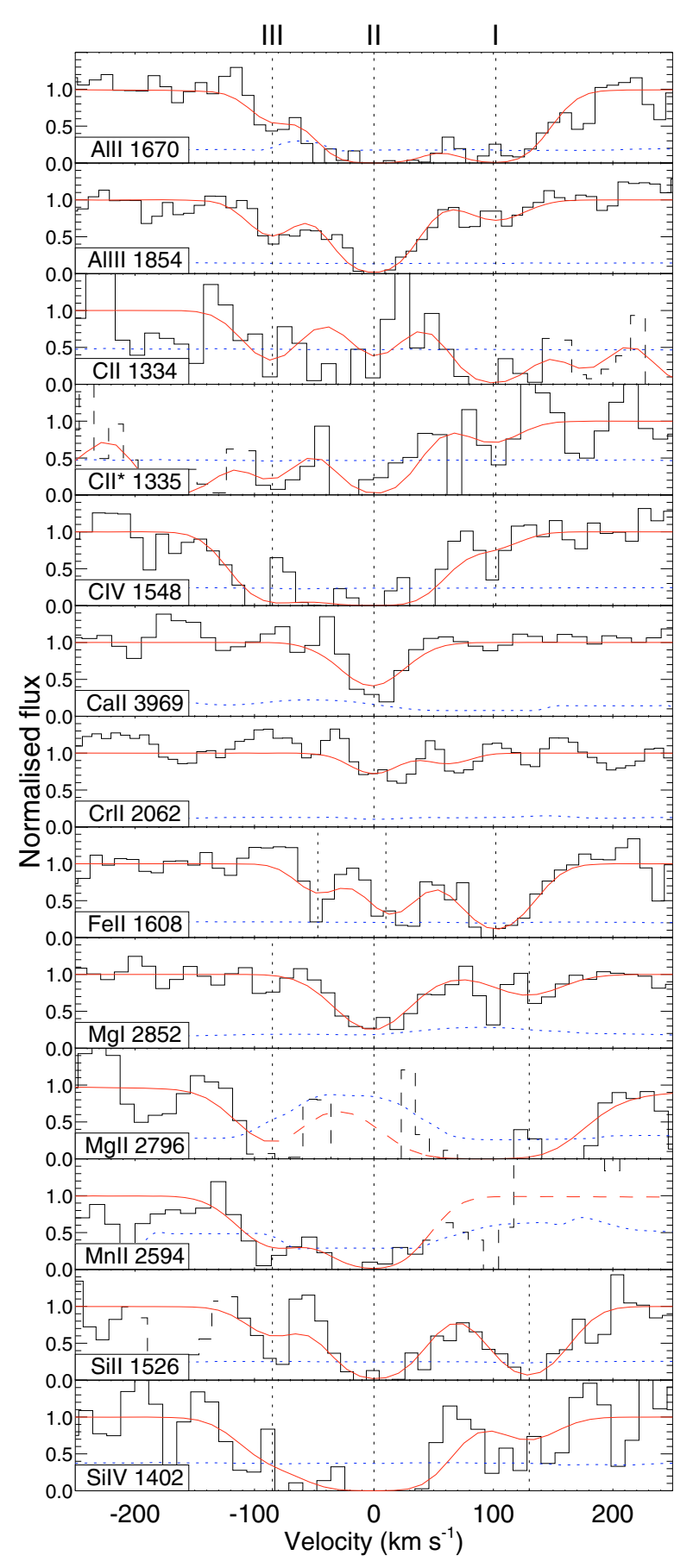

Fig. 3. Fit of the detected of the detected transitions at the GRB redshift. For the elements with several lines detected, we show the transitions that are least saturated or have the highest $\mathrm{S} / \mathrm{N}$. The regions of the spectrum that are excluded from the fit because of the contamination by sky lines or other unrelated absorption features are drawn with a dashed line. We indicate the error spectrum with a dotted (blue) line. Vertical dotted (black) lines mark the different velocity components. See the electronic edition for a colour version of this figure.

From the values in Table 2 and from Fig. 3 it is inferred that component II has the highest column densities, so we used it to calculate the redshift. Component I is the least ionised (high ionisation lines are only weakly detected), while in component III we mainly detect highly ionised species. Although not conclusive, this could indicate that component III is the closest to the

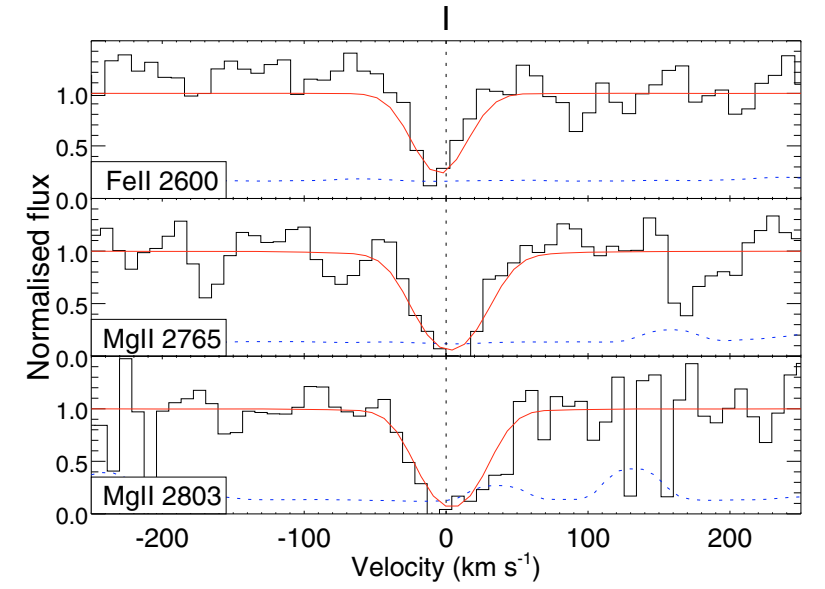

Fig. 4. Fit of $\mathrm{Mg}$ II and Fe II lines of the intervening system at $z=$ 1.9597 .

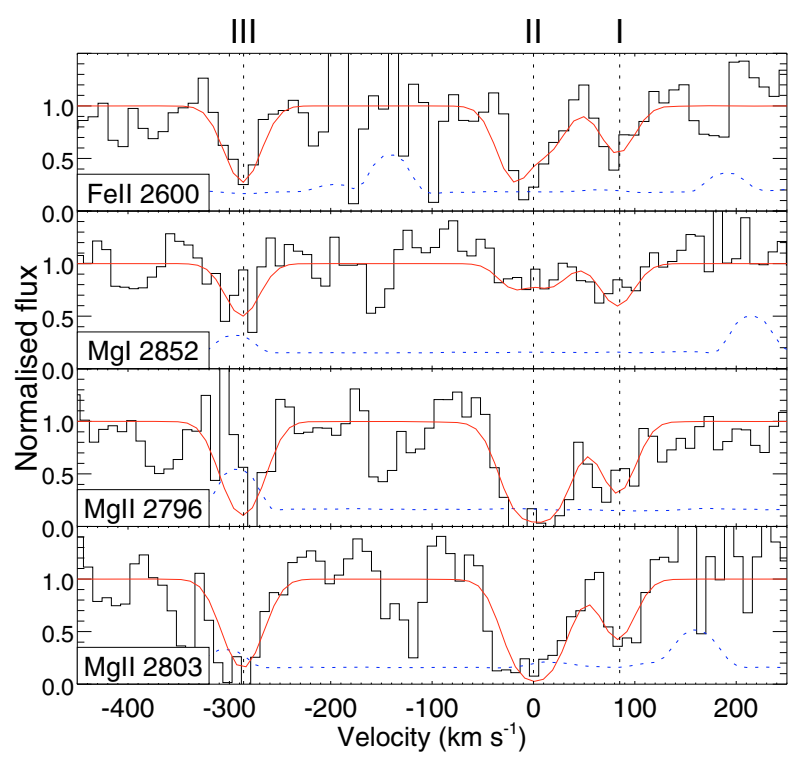

Fig. 5. Fit of $\mathrm{Mg}$ I, $\mathrm{Mg}$ II, and Fe II lines of the intervening system at $z=1.8005$, with three velocity components.

GRB, while component I is the furthest. The relative velocities between the 3 different components come from the relative motion of the gas within the host galaxy, in principle unrelated to the GRB itself. The high values of $[\mathrm{Si} / \mathrm{Fe}]$ measured in the GRB host galaxy may be indicative of enhanced abundance of Si (produced by extensive star formation) or a higher depletion level of $\mathrm{Fe}$ (evidence of dust). In fact, Kann et al. (2009) measure an extinction corresponding to $A_{V}=0.34 \pm 0.15$ for this burst, which as they note, is atypically high for such a high redshift event. They also note that GRB 090313 is among the most intrinsically luminous optical afterglows detected. However, if we look at the $E W_{\text {rest }}$ measured for the absorption features in this spectrum (see Table 1), we find that they are consistent with the typical values found in the sample of Fynbo et al. (2009). We note that, to our knowledge, some features such as $\mathrm{Mg}$ II, Mg I and Ca II had not been observed before in GRB spectra at such high redshift.

The surveys of strong $\mathrm{Mg}$ II $\left(E W_{\text {rest }}>1 \AA\right)$ intervening absorbers along GRB lines of sight gave the surprising result of an excess of these systems compared to QSO lines of sight (Prochter et al. 2006; Vergani et al. 2009). We measured the $E W_{\text {rest }}$ of the $\mathrm{Mg}$ II $\lambda 2796$ line of both intervening systems, finding a combined value (coadd of the three components) of $1.35 \pm 0.10 \AA$ for the $z=1.80$ and $0.50 \pm 0.03 \AA$ for the $z=1.96$. 
The number density of strong Mg II systems for this spectrum is $\mathrm{d} n / \mathrm{d} z=0.88$, in agreement with the excess of a factor of $\sim 2$ compared to the QSO lines of sight found by Vergani et al. (2009). The same authors also considered the statistics of weak systems $\left(0.3 \AA<E W_{\text {rest }}<1 \AA\right)$ and conclude that their number density agrees with what is found along QSO lines of sight, as confirmed by Tejos et al. (2009). The S/N of the GRB 090313 $\mathrm{X}$-shooter spectrum is too low to cover a significant redshift path for a rest frame equivalent width limit of $0.3 \AA$; on the other hand, this limit will be normally reached for the guaranteed time programme for observations of GRB afterglows that will be performed with $\mathrm{X}$-shooter. This programme will collect about 100 spectra within 3 years, hugely increasing the redshift path of the GRB surveys, hence the significance of the statistics, and bringing useful information to explain the unexpected excess of strong absorbers. Moreover, X-shooter will simultaneously cover a longer redshift path compared to the instruments presently used for QSO and GRB afterglow spectroscopic observations, up to $\Delta z \sim 5$ (the total redshift path for strong $\mathrm{Mg}$ II systems for the spectrum presented here is $\Delta z=2.1$ ). Therefore $\mathrm{X}$-shooter QSO and GRB surveys will be able to systematically investigate the presence and nature of $\mathrm{Mg}$ II systems up to a much higher redshift than currently done.

No significant emission lines have been detected at the redshift of the GRB or in any of the intervening systems. Berger (2009) points out the presence of two galaxies near the afterglow (G1 and G2, see Fig. 1). The brightest one (G1, $r=15.6$ ) has a known redshift of $z=0.0235$ and is located at 17 '. 8 from the afterglow, equivalent to $8.3 \mathrm{kpc}$ projected at the redshift of the galaxy. We do not see any features at this redshift and, in particular, nothing is detected at the wavelength of the most prominent absorption features that can be seen in the SDSS spectrum of G1 (Abazajian et al. 2009), which are the Ca II $\lambda 8500$, 8544, 8664, and $\mathrm{Na} \mathrm{I} \lambda 5891,5897$. The $\mathrm{Ca}$ II features are not detected with 3$\sigma$ limits of $E W_{\text {rest }}<0.6 \AA$ and Na I with limits of $E W_{\text {rest }}<1.2 \AA$. The closest galaxy, G2 at 2'. 3, with $r=21.6$ does not have an identified redshift, so its relation with any of the observed absorption features will need further investigation.

To conclude, we point out some facts that show X-shooter's potential in the GRB field. Given a 10- $\sigma$ limiting magnitude (per spectral bin) of $R \sim 21.0$ with $1 \mathrm{~h}$ exposure, it will be able to study $33 \%(72 \%)$ of the long bursts 12 (2) h after the onset (using optical fluxes and decay indices from Nysewander et al. 2009). As an example, X-shooter would have been able to obtain a spectrum similar to or better than the one presented here of the $z \sim 8.2$ GRB 090423 (Tanvir et al. 2009; Salvaterra et al. 2009) during the first $24 \mathrm{~h}$ and to follow the nearby GRB 030329 for over a month. If we consider short GRBs, we expect to be able to study $3 \%(17 \%)$ within 12 (2) h of the burst onset. Together with the rapid response mode available at VLT, which allows reaction times of just a few minutes, the possibilities increase. With its intermediate resolution, we will distinguish components with differential velocities of $\sim 30 \mathrm{~km} \mathrm{~s}^{-1}$. As shown here, this resolution will allow abundances of the different element species to be derived through line fitting, although with some limitations as compared to higher resolution spectrographs when fitting blended components or marginally saturated lines. Thanks to the wide wavelength coverage, it will be capable of observing afterglows up to redshifts of $\sim 18$, assuming that they exist (at redshift 18 , Ly- $\alpha$ would lie at $\sim 23000 \AA$ ). It will allow systematic studies of lines over wide redshift ranges ( $\mathrm{Mg}$ II $\lambda 2796,2803$ will be detectable from $z=0.1$ up to $z=7.5$ ), as well as measurements of ratios with widely separated lines. Furthermore, through the wide spectral coverage, we will have more lines to determine metallicities with better accuracy. It will also allow study of dust extinction profiles in GRB environments. Thanks to the increased sensitivity compared to other instruments of similar resolution, we will be able to study feature variability with higher time resolution and up to later times. All this places $\mathrm{X}$-shooter in a position to lead breakthrough advances in GRB research in the next years.

Acknowledgements. We dedicate this paper to the memory of Roberto Pallavicini, who strongly contributed to the success of X-shooter, but prematurely passed away before its first light. A.d.U.P. acknowledges support from an ESO fellowship. This work is supported by ASI grant SWIFT I/011/07/0, by the Ministry of University and Research of Italy (PRIN MIUR 2007TNYZXL). The Dark Cosmology Centre is funded by the Danish National Research Foundation. We thank the anonymous referee for constructive comments.

\section{References}

Abazajian, K. N., Adelman-McCarthy, J. K., Agüeros, M. A., et al. 2009, ApJS, 182,543

Asplund, M., Grevesse, N., \& Sauval, A. J. 2005, in Cosmic Abundances as Records of Stellar Evolution and Nucleosynthesis, ed. T. G. Barnes III \& F. N. Bash, ASP Conf. Ser., 336, 25

Berger, E. 2009, GRB Coordinates Network, 8984

Bloom, J. S., Perley, D. A., Li, W., et al. 2009, ApJ, 691, 723

Chen, H.-W., Perley, D. A., Pollack, L. K., et al. 2009, ApJ, 691, 152

Chornock, R., Li, W., \& Filippenko, A. V. 2009a, GRB Coordinates Network, 8979

Chornock, R., Perley, D. A., Cenko, S. B., et al. 2009b, GRB Coordinates Network, 8994

Cobb, B. E. 2009, GRB Coordinates Network, 9008

de Ugarte Postigo, A., D’Odorico, S., Vernet, J., et al. 2009a, GRB Coordinates Network, 9015

de Ugarte Postigo, A., Gorosabel, J., Sota, A., \& Castro-Tirado, A. J. 2009b, GRB Coordinates Network, 8992

de Ugarte Postigo, A., Gorosabel, J., Sota, A., et al. 2009c, GRB Coordinates Network, 8999

D’Elia, V., Fiore, F., Perna, R., et al. 2009, ApJ, 694, 332

Dessauges-Zavadsky, M., Chen, H.-W., Prochaska, J. X., Bloom, J. S., \& Barth, A. J. 2006, ApJ, 648, L89

D’Odorico, S., Dekker, H., Mazzoleni, R., et al. 2006, in SPIE Conf. Ser., 6269 Fontana, A., \& Ballester, P. 1995, The Messenger, 80, 37

Fynbo, J. P. U., Jakobsson, P., Prochaska, J. X., et al. 2009, ApJS, 185, 526 Goldoni, P., Royer, F., François, P., et al. 2006, in SPIE Conf. Ser., 6269 Goldoni, P., Royer, F., Horrobin, M., et al. 2008, in SPIE Conf. Ser., 7014 Hurley, K., Boggs, S. E., Smith, D. M., et al. 2005, Nature, 434, 1098 Jakobsson, P., Hjorth, J., Fynbo, J. P. U., et al. 2004, A\&A, 427, 785 Kann, D. A., Masetti, N., \& Klose, S. 2007, AJ, 133, 1187

Kann, D. A., Klose, S., Zhang, B., et al. 2009, ApJ, submitted, [arXiv:0712.2186]

Kouveliotou, C., Meegan, C. A., Fishman, G. J., et al. 1993, ApJ, 413, L101

Mao, J., Margutti, R., Sakamoto, T., et al. 2009, GCN Report, 204

Nakar, E. 2007, Phys. Rep., 442, 166

Nysewander, M., Fruchter, A. S., \& Pe'er, A. 2009, ApJ, 701, 824

Perley, D. A. 2009, GRB Coordinates Network, 8997

Perley, D. A., Cenko, S. B., Bloom, J. S., Li, W., \& Cobb, B. E. 2009a, GRB Coordinates Network, 9001

Perley, D. A., Li, W., Chornock, R., \& Filippenko, A. V. 2009b, GRB Coordinates Network, 8985

Prochaska, J. X., Chen, H.-W., Dessauges-Zavadsky, M., \& Bloom, J. S. 2007, ApJ, 666, 267

Prochter, G. E., Prochaska, J. X., Chen, H.-W., et al. 2006, ApJ, 648, L93

Racusin, J. L., Karpov, S. V., Sokolowski, M., et al. 2008, Nature, 455, 183

Sakamoto, T., Barthelmy, S. D., Baumgartner, W. H., et al. 2009, GRB Coordinates Network, 8986

Salvaterra, R., Della Valle, M., Campana, S., et al. 2009, Nature, 461, 1258

Tanvir, N. R., Fox, D. B., Levan, A. J., et al. 2009, Nature, 461, 1254

Tejos, N., Lopez, S., Prochaska, J. X., et al. 2009, ApJ, 706, 1309

Thöne, C. C., D’Avanzo, P., Fugazza, D., et al. 2009, GRB Coordinates Network, 9012

Updike, A. C., Klose, S., Clemens, C., \& Greiner, J. 2009, GRB Coordinates Network, 8983

Vergani, S. D., Petitjean, P., Ledoux, C., et al. 2009, A\&A, 503, 771

Vreeswijk, P. M., Ledoux, C., Smette, A., et al. 2007, A\&A, 468, 83

Woosley, S. E., \& Bloom, J. S. 2006, ARA\&A, 44, 507 